\title{
The Bologna Process: How student mobility affects multi-cultural skills and educational quality
}

Lydia Mechtenberg* Roland Strausz**

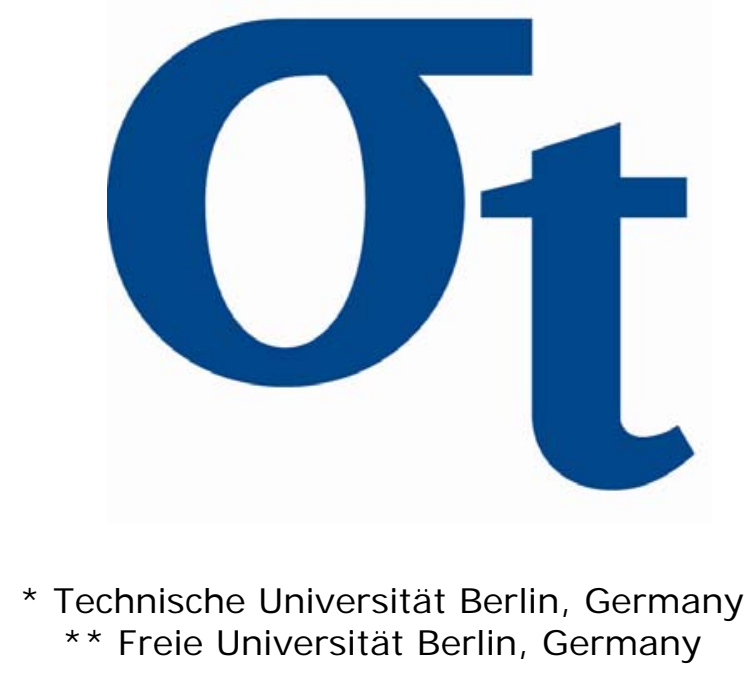




\title{
The Bologna Process: \\ How student mobility affects multi-cultural skills and educational quality
}

\author{
Lydia Mechtenberg and Roland Strausz* \\ Technical University Berlin, Free University Berlin
}

March 2, 2006

\begin{abstract}
We analyze the two goals behind the European Bologna Process of increasing student mobility: enabling graduates to develop multicultural skills and increasing the quality of universities. We isolate three effects: 1) a competition effect that raises quality; 2) a free rider effect that lowers quality; 3) a composition effect that influences the relative strengths of the two previous effects. The effects lead to a trade-off between the two goals. Full mobility may be optimal, only when externalities are high. In this case, student mobility yields inefficiently high educational quality. For moderate externalities partial mobility is optimal and yields an inefficiently low quality of education.
\end{abstract}

Keywords: Student mobility, Quality of higher education, Multi-cultural skills, Bologna Process.

JEL-Codes: D61, H77, I28.

${ }^{*}$ Corresponding author: Lydia Mechtenberg, Technical University Berlin, H50, Strasse des 17. Juni 135, 10623 Berlin, Germany. E-mail: l.mechtenberg@ww.tu-berlin.de. Tel: +49-30-314-29760. We thank Kai Konrad, the participants of the Conference on Higher Education, Multijurisdictionality and Globalisation in Mons for helpful comments and suggestions. Roland Strausz acknowledges support by the DFG (German Science Foundation) under SFB/TR-15 and grant STR-991/1. This research was supported by the Deutsche Forschungsgemeinschaft through the SFB 649 "Economic Risk". 


\section{Introduction}

The so-called Bologna Process belongs to those European policy processes that have attracted considerable public attention. Its intention is to harmonize the diverse European university systems and, thereby, achieve a higher degree of comparability. It is hoped that more comparable university systems lead to higher student mobility. Thus, the Bologna Process can be understood as a process of increasing student mobility. For two reasons, student mobility is seen as key in increasing the productivity of the highly educated. First, student mobility across countries exposes students directly to the different European cultures and helps them to develop their multicultural skills. These skills are seen as indispensable in a European Union that strives for full economic integration while preserving the diversity of its cultures. ${ }^{1}$ Second, increasing student mobility is hoped to kindle a competition between countries to attract the most able students. Since university education in Europe is essentially free, the dimension in which such competition takes place is quality. Hence, it is hoped that higher mobility raises university quality. This would increase the productivity of graduates further.

This paper analyzes the two rationales behind the Bologna Process to increase student mobility: enabling graduates to develop their multi-cultural skills and increasing quality competition between universities. We study how these two goals can be achieved and in how far they are compatible with each other. Thus, we present a framework in which the productivity of students depends both on multi-cultural skills and the quality of university education. Within this setup we compare and evaluate, for different levels of student mobility, the expenditures in higher education that are chosen by two symmetric, benevolent governments.

Our main result is that the claimed competition effect which is supposed to raise quality is rather unlikely to occur; it happens only if the externality generated by foreign students is high enough. Indeed, in order for the competition effect to raise quality, it must overcome the free rider effect that countries prefer their students to obtain their costly education abroad. Only if students are relatively unlikely to return from a foreign education and only if a country is able to appropriate a large share of a foreigner's productivity,

\footnotetext{
${ }^{1}$ See for instance Jahr, et al. (2002, p. 50ff).
} 
does the positive competition effect occur. Yet, for more reasonable values of the students' return probabilities and appropriation of their productivity, the free riding effect outweighs the competition effect. Hence, with respect to the Bologna Process it seems more reasonable to expect that increased student mobility actually lowers university quality.

As a result, student mobility yields a trade-off between an acquisition of multi-cultural skills and the quality of university education. Higher student mobility facilitates the acquisition of multi-cultural skills at the expense of lower investments in higher education. The optimal level of student mobility will depend on the outcome of this trade-off. Essentially, a higher mobility of students is called for when multi-cultural skills are more important. In contrast, if the free riding effect is stronger, less mobility is preferred.

We study how the trade-off varies for different degrees of student mobility. We thereby obtain a non-monotonic relationship; the competition effect is strongest when student mobility is low. The reason for this is that we model student mobility as a process that facilitates information processing. This captures the idea behind the Bologna process that more comparable university systems facilitate the decision process of a student to study abroad. Thus, we interpret higher student mobility as a reduction of the student's information processing ability that is required for making the decision to study abroad. In addition, the student's ability to process information also affects his benefits from university education; more talented students achieve a higher productivity than less able students. As a consequence, a rise in student mobility lowers the talent pool of mobile students. This composition effect causes a decline in the competition effect; with higher mobility it becomes less attractive for a country to attract the students, because their average talent, and therefore their expected productivity, will be lower.

The remainder of the paper is organized as follows. The next section discusses related literature. Section 3 presents the model. Section 4 characterizes the first best optimum. Sections 5 and 7 discuss the extreme cases with student immobility and full student mobility, respectively. Section 8 analyses the properties of the second best optimum for intermediate degrees of student mobility. The final section concludes. All formal proofs are collected in the appendix. 


\section{Related Literature}

The relation between mobility and human capital has for long been on the agenda of economic research. The larger part of the literature that deals with this topic analyses the consequences which increasing mobility of skilled labour might have on human capital investments. The characteristic time structure of these models includes the assumption that individuals have to decide about their educational effort or costs before they eventually become mobile.

Within this framework, the Brain Drain literature identifies two main consequences which increasing mobility of highly productive labour will have on human capital investments. Firstly, as pointed out by Grubel and Scott (1966) and Bhangwati and Hamada (1974) in an asymmetric setting with a pure sender country and a pure receiver country, the sender country will reduce its public investment in education if an increasing fraction of highly educated individuals leaves the country. Similarly, Justman and Thisse (1997) show that in symmetric settings, too, where each country is both sender and receiver country, exogeneous mobility of graduates leads to an underprovision of publicly provided education.

By contrast, Stark et al. (1997), Beine et al. (2001) and Stark and Wang (2002) take also private investments in education into account. They demonstrate that with one poor sender country and one rich receiver country, the reduction in public provision of education in the sender country can be overcompensated by the increase of private investments due to the rising private returns to education if highly skilled labour becomes more mobile.

Poutvaara (2004a) and (2004b) also consider both public and private investment in education. Poutvaara shows that although increasing international applicability of education leads to higher private investments, the governments tend to reduce public funds for internationally applicable education.

Thus, the most stable result that is established by this kind of literature is that although increasing mobility of graduates will lead to higher private investments in education, public provision will decrease. The governments will tend to free ride on the education system of the other country. 
Büttner and Schwager (2004) produce a similar result within a different framework. Their paper belongs to the small part of the literature on education economics that explicitly focuses on the consequences of increasing student mobility. Here, the time structure is different. Students are already mobile when they have to decide about their educational investment; and they are able to choose the country in which they want to study. Büttner and Schwager (2004) show that if students are mobile, welfare states want to free ride on the education system of the other country, so that in equilibrium, public provision of education is too low.

Kemnitz (2005), by contrast, considers not only the free rider effect, but also the competition effect of governments that provide education for mobile students. If students generate positive expected externalities in the country in which they study, the incentive to free ride on the neighbouring country's education system is not the only incentive at work. It may be profitable for the government to raise its funds in order to attract more students. The question which of the two effects prevails depends on the relative importance of the positive externalities and the costs that are generated by students. Kemnitz (2005) compares free rider and competition effect within a framework where the governments are driven by exploitative incentives.

In relation to the existing literature, our focus is on the effects of student mobility on public provision of education with benevolent governments. Our contribution to the existing literature is twofold. First, we compare the free rider effect and the competition effect within a symmetric setting where two welfare states provide higher education for their mobile students. Thus, we show that also if the governments are not driven by exploitative incentives but are welfare states, a competition effect may be identified that may drive public provision of education upwards. Yet, the magnitude of this effect is rather small. Second, we are the first to take into account that student mobility is positively correlated with talent ${ }^{2}$, and that the talent distribution is continuous. This assumption allows us to analyse how the relative weights of free rider effect and competition effect change with increasing student

\footnotetext{
${ }^{2}$ Jahr, et al. (2002, p. 44ff) report that mobility of a graduate is positively correlated with both the education level of the parents and her own mobility as a student. Given a positive correlation between talent and the education level of parents, this finding provides indirect evidence that student mobility and talent are positively correlated.
} 
mobility.

\section{The Setup}

We consider two symmetric countries $A$ and $B$. Each country consists of an infinite number of students with mass one. Students differ in two dimensions. First, they differ in their learning ability $\tau$ which is uniformly distributed over the unit interval $[0,1]$. This learning ability represents a student's general ability to process, learn, and use new and complex information. It influences both the returns from the student's intellectual and multi-cultural training. Second, students are heterogeneous with respect to their multi-cultural skills. Some students acquire higher multi-cultural skills when studying abroad, while others may benefit more from studying at home. Hence, if we represent by $a$ the relative gain in multi-cultural skills from studying abroad, then for those students who benefit more from a foreign education the parameter $a$ is positive, whereas it is negative for other types of students. For tractability, we assume that multi-cultural skills $a$ are uniformly distributed over the interval $[-1 / 2,1 / 2]$ and are independent of learning ability $\tau$. Hence, a student $k$ is characterized by a learning ability $\tau_{k} \in[0,1]$ and multi-cultural skill $a_{k} \in$ $[-1 / 2,1 / 2]$. Students are fully informed about their characteristics and will take them into account when deciding whether or not to study abroad.

Given the student's population, each government $i$ chooses university quality $q_{i}$, with the intention to maximize the welfare of its natives. University quality raises intellectual skills and therefore the productivity of students who graduate from university.

Next, students choose the country in which they want to study. We model mobility by assuming that only students who are above a given talent level $\hat{\tau}$ are mobile. This assumption captures the idea behind the Bologna Process that more comparable universities increase student mobility. This idea follows naturally from the simple fact that students must first learn about foreign university systems before they decide whether to study abroad. With more diverse systems, it is more difficult for a student to acquire and process the relevant information. Hence, we view the Bologna Process as a process that lowers the informational barriers to mobility; more divergent 
university systems represent higher informational barriers than standardized ones. Since the ability to process information depends on the student ability $\tau$, only the more able students $\tau \geq \hat{\tau}$ will find it worthwhile to obtain and process the necessary information for deciding whether to study abroad.

A student's return from a university education depends on the university's quality of education $q_{i}$. In particular, a student $k$ who is born in country $i$ with learning ability $\tau_{k}$ and multi-cultural skills $a_{k}$ attains a productivity of

$$
\pi_{k i}=\tau q_{i}
$$

if she studies at home. If, instead, she studies abroad, her productivity depends on the foreign university quality $q_{j}$. In addition, her productivity is affected by her multi-cultural skill $a_{k}$. Thus, a foreign study yields a productivity of

$$
\pi_{k j}=\tau\left[q_{j}+m a_{k}\right] .
$$

The parameter $m \geq 0$ thereby measures the relative importance of multicultural skills. If these skills are unimportant, then $m=0$. Otherwise, $m>0$.

Whether or not a mobile student migrates depends solely on how much she will gain if she graduates at the foreign university, compared with her gain from studying in her home country. We assume that the graduate appropriates only a fraction $\gamma<1$ of her productivity $\pi$. The remaining fraction $(1-\gamma)$ is appropriated by the country in which the graduate works. There are no direct migration costs. Consequently, a student $k$ who has been born in country $i$ with multi-cultural skill $a_{k}$, will migrate if and only if

$$
\gamma \pi_{k i} \geq \gamma \pi_{k j} \Rightarrow q_{j}+m a_{k}>q_{i}
$$

Hence, given quality levels $q_{i}$ and $q_{j}$, a student with multi-cultural skills $\bar{a}=\left(q_{i}-q_{j}\right) / m$ is indifferent about studying abroad. Consequently, all students with multi-cultural skills $a>a_{0}^{i}$ have an incentive to study abroad, where ${ }^{3}$

$$
a_{0}^{i}=\max \left\{-1 / 2, \min \left\{1 / 2,\left(q_{i}-q_{j}\right) / m\right\}\right\} .
$$

\footnotetext{
${ }^{3}$ The maxmin expression takes account of possible corner solutions.
} 


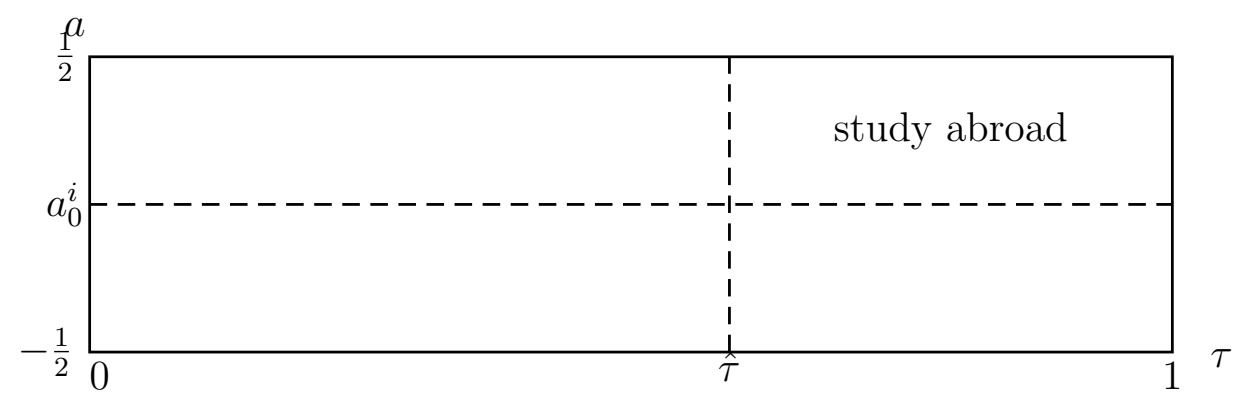

Figure 1: Students

Figure 1 illustrates the share of students who study abroad. Since students are uniformly distributed, the number of students from country $i \in$ $\{A, B\}$ who study abroad is

$$
n_{i j}=\left(\frac{1}{2}-a_{0}^{i}\right)(1-\hat{\tau}) .
$$

The remaining $n_{i i}=1-n_{i j}$ students study at home.

After students complete their studies, they start working and receive a share $\gamma$ from their productivity. The remaining share $1-\gamma$ is appropriated by the country where the student works. We assume that students who studied at home are immobile and therefore work in their home country. Students who studied abroad return to their home country with an exogenous probability $\rho \in[0,1] .{ }^{4}$ A share $1-\rho$ remains in the foreign country. These students will generate a positive externality of a magnitude $(1-\gamma)$ on the foreign country.

Countries choose their university quality, $q_{i}$, in order to maximize the social welfare of its natives. Social welfare consists of four different components: 1) the contribution to welfare of those students who study at home, $\left.W_{i i}^{i} ; 2\right)$ the contribution of a country's students who study abroad, $W_{i j}^{i} ; 3$ ) the contribution of foreign students who stay working in the country after completing their studies, $W_{j i}^{i}$; and 4$)$ the costs that are generated by the total sum of students studying at its university, $C_{i}^{i}$.

\footnotetext{
${ }^{4}$ The assumption captures the empirical fact (e.g., Jahr et al. 2002) that mobility before and after graduation are positively correlated; mobile students are more likely to emigrate than students who studied at home.
} 
A country appropriates the entire surplus of its own students that study at home. As illustrated in Figure 1, there exist two types of students that study at home: first, those students $a<a_{0}^{i}$ who do not benefit from a foreign education and, second, those students that, due to limited access to information, are unable to study abroad. Since a home student of ability $\tau$ obtains a productivity of $\tau q_{i}$, their contribution to welfare is

$$
W_{i i}^{i}=\int_{-\frac{1}{2}}^{a_{0}^{i}} \int_{0}^{1} \tau q_{i} d \tau d a+\int_{a_{0}^{i}}^{\frac{1}{2}} \int_{0}^{\hat{\tau}} \tau q_{i} d \tau d a .
$$

The contribution to welfare from students that study abroad will depend on the number of students that return after their studies. Country $i$ benefits from the full productivity $\tau\left(q_{j}+m a\right)$ of these students. But since a student appropriates only a share $\gamma$ of his productivity, a student $(\tau, a)$ who not only studies but also stays abroad contributes only $\gamma \tau\left(q_{j}+m a\right)$ to his country's welfare. Hence, the contribution of students that study abroad is

$$
W_{i j}^{i}=\int_{a_{0}^{i}}^{\frac{1}{2}} \int_{\hat{\tau}}^{1}[\rho+(1-\rho) \gamma] \tau\left(q_{j}+m a\right) d \tau d a .
$$

Finally, a country appropriates a share $(1-\gamma)$ of the productivity of foreign students who, after their studies, remain in the country. The contribution of these foreign students to social welfare is

$$
W_{j i}^{i}=\int_{a_{0}^{j}}^{\frac{1}{2}} \int_{\hat{\tau}}^{1}(1-\rho)(1-\gamma) \tau\left(q_{i}+m a\right) d \tau d a
$$

The provision of quality is costly. The form of the cost function depends on whether university quality is considered a public or a private good. If it is a public good, the costs of providing a given quality level are independent on the number of students who consume this quality. If it is a private good, costs per quality increase with the number of consumers. Both assumptions can be found in the literature. Therefore, we assume that university quality has characteristics of both a public and a private good. In particular, country $i$ 's costs from providing quality $q_{i}$ are

$$
C_{i}=k_{1} q_{i}^{2}+k_{2} q_{i}\left(n_{i i}+n_{j i}\right)
$$


The importance of the public good's and private good's aspect of quality are measured by the parameters $k_{1}$ and $k_{2}$ respectively.

To summarize, country $i$ 's social welfare, $W^{i}$, is the sum of the individual components,

$$
W^{i}=W_{i i}^{i}+W_{i j}^{i}+W_{j i}^{i}-C_{i}^{i}
$$

Aggregate social welfare is

$$
W=W^{A}+W^{B}
$$

\section{The First Best}

If countries were able to coordinate their quality decision, they would agree to implement the efficient level that maximizes aggregate social welfare $W$. The following proposition derives the efficient quality level.

Proposition 1 The efficient level of quality is independent of the mobility parameter $\hat{\tau}$ and is $q_{A}=q_{B}=q_{E}$, where

$$
q_{E} \equiv \frac{1-2 k_{2}}{4 k_{1}}
$$

As expected, the efficient level is decreasing in the cost parameters $k_{1}$ and $k_{2}$. The efficient level, $q_{E}$, does not depend on $\gamma$ and $\rho$, because these parameters only determine how the graduate's productivity is shared between the two countries. In the aggregate, the division of rents is irrelevant. Finally, the proposition shows that the efficient quality level is independent of student mobility $\hat{\tau}$. We obtain this independence result, because intellectual and multi-cultural skills are additive separable components of the graduate's productivity. It implies that, from a social welfare perspective, there is no trade-off between quality and mobility.

In a first best world, benevolent governments would not only implement the efficient quality level of university education, but also choose an efficient degree of student mobility. Since from an aggregate perspective there is no trade-off between quality and mobility and because students take efficient studying decisions, it is optimal to enable all students to develop their multicultural skills. The next proposition formalizes this result. 
Proposition 2 If $m=0$, any degree of student mobility is consistent with the first best optimum. For $m>0$, only full mobility $\hat{\tau}=\hat{\tau}^{*} \equiv 0$ is efficient.

In a first best world, quality levels are set at $q_{A}=q_{B}=q_{E}$ and students enjoy full mobility when multi-cultural skills matter $(m>0)$. When these skills do not matter, then also mobility does not play a role.

\section{$5 \quad$ Immobile Students}

Without the possibility of coordination, each country chooses $q_{i}$ independently with the intention to maximize the social welfare $W_{i}$ of its natives. This may lead to distortions, because countries will only consider the effect of their decisions on the social welfare of its natives. Yet, a special case obtains when students are fully immobile $(\hat{\tau}=1)$. For this extreme, there is no interaction between the countries. Each country incurs all the costs from educating its citizens and also fully benefits from their productivity. Consequently, countries choose the first best level of university quality.

Thus, restricting student mobility has the advantage that it yields efficient investment decisions $q_{E}$. A disadvantage is however that some students are unable to develop their multi-cultural skills. Hence, in the case that such skills matter $(m>0)$, the first best level of university quality comes at the expense of inefficient cultural skills. The following proposition makes this intuition more precise.

Proposition 3 If $\hat{\tau}=1$, the countries choose the efficient quality levels $q_{A}^{*}=q_{B}^{*}=q_{E}$. The first best optimum is reached only if $m=0$.

Hence, full efficiency is reached non-cooperatively when multi-cultural skills do not matter. This result provides an initial justification for leaving the choice of university quality in the hands of the member states of the European Union. But as will be shown, no such justification is possible when multi-cultural skills become part of human capital. As the European Union's economic integration progresses, multi-cultural skills become more important and a total restriction on student mobility forecloses potential gains from productivity. 


\section{Mobile Students}

When multi-cultural skills matter $(m>0)$, some graduates may increase their productivity by developing their multi-cultural skills abroad. This yields an efficiency argument in favor of student mobility. This section analyzes this argument in closer detail.

Without coordination, each country chooses its university quality $q_{i}$ with the goal to maximize its social welfare $W^{i}$. Whenever $\hat{\tau}<1$, its optimal quality choice depends on the choice of the neighboring country. Hence, there exist strategic interactions. The following lemma derives the Nash equilibrium levels that result from the strategic interaction.

Lemma 1 Suppose multi-cultural skills matter $(m>0), k_{1}>1 / m$, and $k_{2}<1 / 4 .^{5}$ Then there exists a unique Nash equilibrium $\left(q_{A}^{*}, q_{B}^{*}\right)$ in pure strategies. The Nash equilibrium is symmetric so that $q_{A}^{*}=q_{B}^{*}=q^{*}$ with

$$
q^{*} \equiv \frac{\left(1-4 k_{2}+\hat{\tau}^{2}+\left(1-\hat{\tau}^{2}\right) E\right) m}{8 m k_{1}+8 k_{2}(1-\hat{\tau})-4\left(1-\hat{\tau}^{2}\right) E}
$$

and

$$
E \equiv(1-\rho)(1-\gamma)
$$

The lemma shows that, in general, the equilibrium level $q^{*}$ differs from the efficient level $q_{E}$. Countries' non-cooperative investment decisions are distorted away from the first best, because at the individual level countries do not take into account the effect of their decisions on their neighbors. From the equilibrium levels (3) we may identify two effects that distort choices: a competition and a free rider effect. Since our main results depend on the interplay of these two effects, we discuss them briefly before continuing our analysis.

First, when foreign students remain in the hosting country after their studies, the hosting country appropriates a part of their productivity. Consequently, foreign students exert a positive externality on the social welfare

\footnotetext{
${ }^{5}$ The condition $k_{1}>1 / m$ guarantees that the social welfare function $W^{i}$ is concave in $q_{i}$ for all parameters values of $(\gamma, \rho, \tau)$. Moreover, the reaction functions are falling for all parameter values $(\gamma, \rho, \tau)$ whenever $k_{2}<1 / 4$.
} 
of a country. The size of the externality is proportional to $E=(1-\rho)(1-\gamma)$, because foreign students stay with probability $1-\rho$ and, in this case, the hosting country appropriates a share $1-\gamma$ of their productivity. The positive externality gives a country an incentive to attract foreign students. At the same time, countries expect to lose a share $E$ from the productivity of their students who study abroad. The externality $E$ therefore also measures the incentive of a country to induce its natives to study at home. In the aggregate, the parameter $E$ measures the overall incentives of countries to raise the quality of education in order to attract foreign students and keep their own students from studying abroad. Thus, $E$ is a measure of the competition effect of student mobility that is claimed in the policy debates surrounding the Bologna process.

The equilibrium levels (3) also reveal that quality choices are distorted even when there is no competition effect $E=0$. This is due to a negative free riding effect. If $\hat{\tau}<1$ countries may be tempted to educate their students abroad in order to save on the cost of education. This option is especially attractive if there does not exist a competition effect $(E=0)$. In this case, there is no loss attached to educating one's students abroad. Because a country saves the cost $k_{2}$ on each individual that is educated abroad, the free riding effect is also stronger when the private good's aspect of education, $k_{2}$, is larger.

Hence, whenever students are mobile $(\hat{\tau}<1)$ the countries' investment choices depend on the interplay between the competition effect and the free rider effect. The remainder of this paper takes a closer look at how student mobility affects this interplay.

\section{$7 \quad$ Full Mobility}

Section 5 explained that the disadvantage of immobility $(\hat{\tau}=0)$ is that student's cannot fully develop their multi-cultural skills. This section focuses on the other extreme of full mobility $(\hat{\tau}=0)$, where students are able to realize their multi-cultural skills to their full potential. Yet, as may be expected, this extreme generally leads to inefficient quality choices. Depending on the relative strengths of the competition and free rider effect, these quality choices will be either inefficiently low or inefficiently high. 
Proposition 4 At full mobility $(\hat{\tau}=0)$ the equilibrium level of university quality is

$$
q_{A}^{*}=q_{B}^{*}=q^{*}=\frac{\left(1-4 k_{2}+E\right) m}{8 m k_{1}+8 k_{2}-4 E} .
$$

For $E=E_{H}$, the equilibrium is efficient. For $E<E_{H}$ equilibrium levels are inefficiently low. For $E>E_{H}$ equilibrium levels are inefficiently high, where

$$
E_{H} \equiv \frac{m k_{1}+2 k_{2}\left(1-2 k_{2}\right)}{m k_{1}+1-2 k_{2}} .
$$

The proposition shows that only for the non-generic case $E=E_{H}$ full mobility leads to a first best efficient outcome. In this special case, the positive competition effect and the negative free riding effect balance each other out exactly. Yet, in general we will have $E \neq E_{H}$ and, depending on whether the students' externality is smaller or larger than $E_{H}$, full mobility leads to either inefficiently low quality levels or inefficiently high quality levels. Hence, when multi-cultural skills matter $(m>0)$ and $E \neq E_{H}$, the first best cannot be obtained. The two goals of Bologna of maximizing multicultural skills and, at the same time, inducing efficient levels of university quality are not fully compatible. In general, there exists a trade-off between the two. The next section studies this trade-off in closer detail.

\section{Intermediate Student Mobility}

Section 5 showed that the extreme of fully immobile students $(\hat{\tau}=1)$ induces efficient university quality choices, but leads to an inefficient development of multi-cultural skills. In contrast, Section 7 obtained that the other extreme of full mobility $(\hat{\tau}=0)$ leads to efficient development of skills, but inefficient quality choices. These results prompt us to study intermediate level of student mobility.

In this section we show how the relative strength of the competition and free rider effect vary with varying levels of student mobility. Moreover, we study what levels of student mobility may be optimal from a second best perspective which optimally trades off the effect that student mobility has 


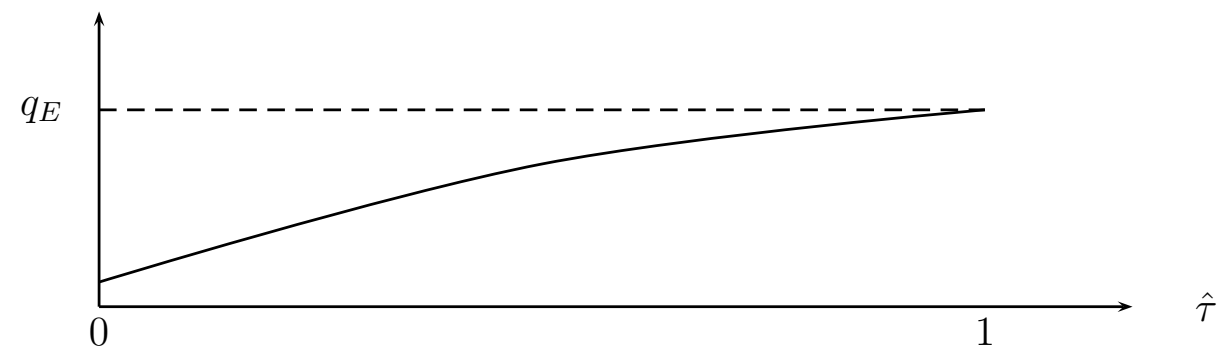

Figure 2: Curve $q^{*}(\hat{\tau})$ for $E<E_{L}$

on the choice of university quality and the development of multi-cultural skills.

The previous section already indicated that the direction in which the quality choices are distorted depends on the relative weights of the competition and free rider effect. In particular, when the free rider effect dominates, the two countries choose inefficiently low quality levels. Clearly, when the dominance of the free rider effect does not depend on student mobility, we obtain a downward distortion for all levels of student mobility. The following lemma shows that this occurs exactly when the externality $E$ is smaller than a critical threshold

$$
E_{L} \equiv \frac{m k_{1}+k_{2}\left(1-2 k_{2}\right)}{m k_{1}+1-2 k_{2}}
$$

Lemma 2 Suppose $E<E_{L}$. Then the Nash equilibrium university quality level $q^{*}(\hat{\tau})$ is increasing in $\hat{\tau}$.

The lemma demonstrates that, for small externalities $\left(E<E_{L}\right)$, the free rider effect dominates the competition effect for all positive levels of student mobility. As illustrated in Figure 2 this implies the following monotonic relationship between university quality and student mobility. When students are immobile $(\hat{\tau}=1)$, the countries choose an efficient level of quality, $q_{E}$. But due to the dominating free rider effect, an increase of student mobility by lowering $\hat{\tau}$ induces countries to lower their quality of education. The downward distortion is strongest for full mobility $(\hat{\tau}=0)$.

The lemma further implies that with low externalities there exists a tradeoff between fostering multi-cultural skills and ensuring an optimal quality of 
education. The following proposition formalizes that the second best solution will be a compromise and leads to an underprovision of quality.

Proposition 5 If $E<E_{L}$, the second best optimum will be characterized by incomplete student mobility $\hat{\tau} \in(0,1)$ and underprovision of university quality $q^{*}<q_{E}$.

When externalities are low, there exists a monotonic relationship between quality and student mobility. This raises the question, whether such a montonic relationship also exists for other levels of externalities. The following lemma shows, however, that for externalities which exceed the level $E_{L}$, the monotonic relationship is lost. In particular, the relationship between $q^{*}$ and $\hat{\tau}$ is positive at $\hat{\tau}=0$ and negative at $\hat{\tau}=1$. Hence, when we regard quality $q^{*}$ as a function of student mobility $\hat{\tau}$, then the curve $q^{*}(\hat{\tau})$ is increasing for small $\hat{\tau}$ and decreasing for larger values of $\hat{\tau}$.

Lemma 3 University quality $q^{*}(\hat{\tau})$ is increasing at $\hat{\tau}=0$ for all E. University quality $q^{*}(\hat{\tau})$ is decreasing at $\hat{\tau}=1$ if and only if $E>E_{L}$.

Proposition 4 demonstrated that with full mobility $(\hat{\tau}=0)$ quality choices are inefficiently low whenever $E<E_{H}$. This result together with Lemma 3 implies that for intermediate values $E \in\left(E_{L}, E_{H}\right)$ the relationship between quality and mobility is as illustrated in Figure 3. For lower values of $\hat{\tau}$, the free riding effect dominates and quality is inefficiently low. For larger values of $\hat{\tau}$ the competition effect dominates and university quality levels are inefficiently high. As illustrated in Figure 3, there exists a threshold value $\bar{\tau}$ at which the free rider and the competition effect cancel each other out, resulting in an efficient level of quality $q_{E}$. The following lemma formalizes this reasoning.

Lemma 4 If $E_{L}<E<E_{H}$, the university quality $q^{*}(\hat{\tau})$ is increasing at $\hat{\tau}=0$ and decreasing at $\hat{\tau}=1$. There exists a unique $\bar{\tau} \in(0,1)$ with $q^{*}(\bar{\tau})=$ $q_{E}$. The derivative of $q^{*}(\hat{\tau})$ is continuous.

Intuitively, the non-monotonic relationship between quality distortion and student mobility is due to the composition effect. With increasing student mobility, the average talent of students who study abroad decreases. 


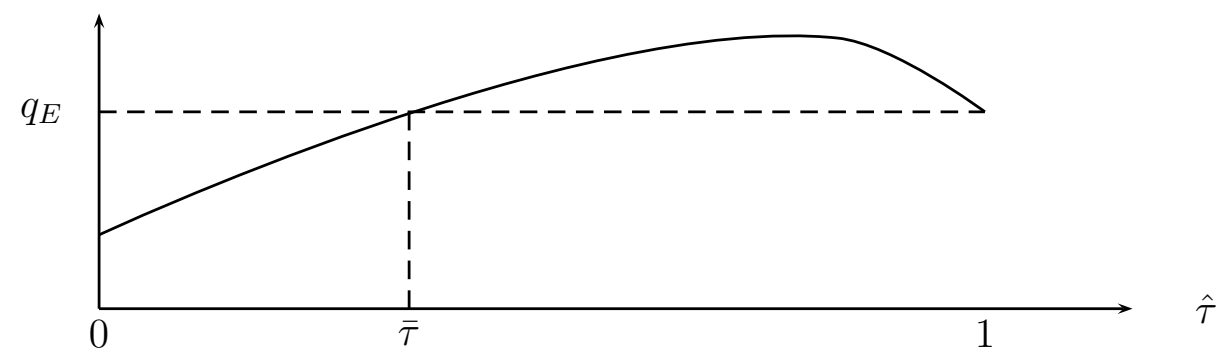

Figure 3: Curve $q^{*}(\hat{\tau})$ for $E_{L}<E<E_{H}$

Consequently, higher mobility reduces the average gain in productivity from these students. In addition, the average talent of foreign students also declines with higher student mobility. As a result, it becomes less attractive to attract these students, because the average productivity gain from them will be lower. Since the costs of education are independent of a student's quality, the reduction in the average talent of mobile students reduces the importance of the competition effect relative to the free rider effect. This is exactly what happens for intermediate values of $E$. In this case, the competition effect is only strong enough to outweigh the free rider effect when the average talent of the mobile students is high.

We now study the optimal second best student mobility $\hat{\tau}^{*}$ with intermediate externalities. First, note that for $\hat{\tau}>\bar{\tau}$ there is no meaningful trade-off between quality and student mobility. A level of mobility $\hat{\tau}=\bar{\tau}$ leads to both a more efficient acquisition of multi-cultural skills and a more efficient quality of education than any level $\hat{\tau}>\bar{\tau}$. As a consequence, the optimal level of student mobility lies below $\bar{\tau}$ and leads to an underprovision of educational quality. This is formalized by the following proposition.

Proposition 6 For $E_{L}<E<E_{H}$, the second best optimum $\hat{\tau}^{*}$ will be smaller than $\bar{\tau}$ and larger than zero. It induces an underprovision of university quality $q^{*}<q_{E}$.

Proposition 6 is the counterpart of Proposition 5 for intermediate externality levels. Both propositions yield qualitatively equivalent results. For both low and intermediate externality levels, neither full mobility, $(\hat{\tau}=0)$, nor full immobility, $(\hat{\tau}=1)$, is optimal. In contrast, the optimal second 
best level of student mobility is intermediate, $\left(\hat{\tau}^{*} \in(0,1)\right)$, and yields an underprovision of university quality.

Finally, we address the possibility that mobile students exercise a considerably high externality on their hosting country $\left(E>E_{H}\right)$. According to Lemma 3 quality is decreasing in the degree of student mobility for low levels of student mobility and increasing for higher levels. This result reflects again the composition effect. For low levels of mobility the average talent of the mobile student is high and the competition effect outweighs the free rider effect. As student mobility increases, the average talent of the mobile student decreases and the free riding effect becomes relatively more important. Yet, for $E>E_{H}$ the competition is so strong that it remains to outdo the free rider effect even at the extreme of full mobility $(\hat{\tau}=0)$. This result is formalized in the following lemma.

Lemma 5 If $E>E_{H}$, university quality $q^{*}(\hat{\tau})$ is increasing at $\hat{\tau}=0$ and decreasing at $\hat{\tau}=1$. Moreover, $q^{*}(\hat{\tau})>q_{E}$ for all $\hat{\tau} \in[0,1)$. The curve $q^{*}(\hat{\tau})$ obtains a unique maximum at $\tilde{\tau} \in(0,1)$ with $q^{*}(\tilde{\tau})>q_{E}$. There exists a unique $\tau^{\prime} \in(\tilde{\tau}, 1)$ such that $q^{*}\left(\tau^{\prime}\right)=q^{*}(0)$.

Figure 4 illustrates the lemma graphically. The curve $q^{*}(\hat{\tau})$ is decreasing for $\hat{\tau}>\tilde{\tau}$. This reflects the range where the competition effect outweighs the free rider effect. Yet, for lower $\hat{\tau}$ the composition effect reduces the relative strength of the competition effect. At $\hat{\tau}=\tilde{\tau}$ the free rider effect is gaining the overhand, and a further reduction in $\hat{\tau}$ reduces the distortion in quality. Yet, for $E>E_{H}$ the initial competition effect is too strong for the free rider effect to eliminate the upward distortion in quality and reverse it. Hence, the curve exceeds the efficient level $q_{E}$.

Finally, we address the second best level of student mobility $\hat{\tau}^{*}$. For the range $\left(0, \tau^{\prime}\right]$ there exists no meaningful trade-off between quality of education and student mobility. In comparison to any level $\hat{\tau} \in\left(0, \tau^{\prime}\right]$, full mobility $(\hat{\tau}=0)$ leads to both a more efficient acquisition of multi-cultural skills and a more efficient quality of education. As a consequence, the optimal level of student mobility lies either at $\hat{\tau}=0$ or $\hat{\tau} \in\left(\tau^{\prime}, 1\right)$. Hence, for high externalities, the strong competition effect leads to an overprovision of educational quality. 


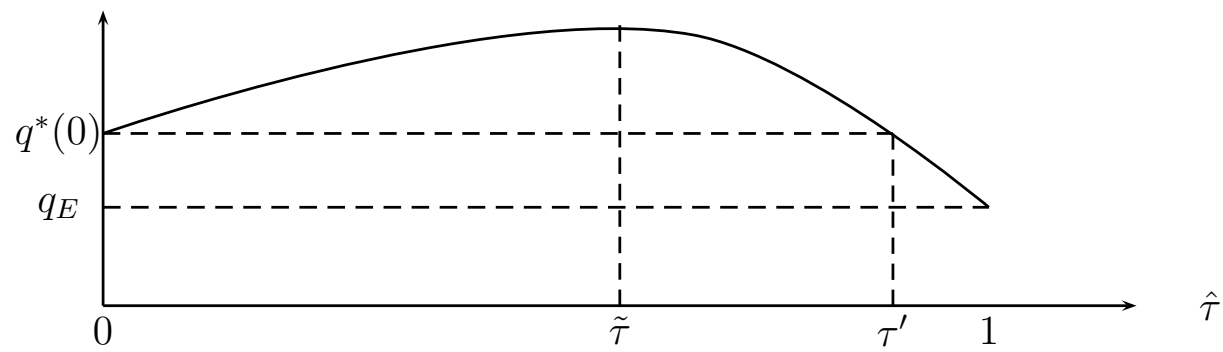

Figure 4: Curve $q^{*}(\hat{\tau})$ for $E>E_{H}$

Proposition 7 If $E>E_{H}$, the second best optimum is characterized by an overprovision of university quality $q^{*}>q_{E}$ and either full student mobility $\hat{\tau}=0$ or positive but incomplete student mobility $\hat{\tau}^{*} \in\left(\tau^{\prime}, 1\right)$.

A comparison of Proposition 7 to Propositions 5 and 6 reveals that qualitative results for large externalities $E>E_{H}$ differ from those obtained for smaller externalities $E<E_{H}$. In particular, we obtain an overprovision of university quality for $E>E_{H}$ against an underprovision of university quality for $E<E_{H}$.

\section{Conclusion}

The Bologna Process is meant to increase student mobility, which, in its turn, is to raise the productivity of European graduates due to two effects. First, mobility is supposed to kindle competition among the union's member states to attract the best students. This competition effect induces governments to raise the quality of their universities. At the same time, higher mobility is expected to provide graduates with higher multi-cultural skills. These skills are believed to become more and more important in a union that strives for economic integration while preserving the diversity of its cultures. The current paper examines the economic rationale behind these arguments. It studies the economic effects of student mobility on both the acquisition of multi-cultural skills and the quality of university education.

We obtain a trade-off between multi-cultural skills and university quality. When students are fully immobile, there is no interaction between countries and governments choose the efficient level of university quality; but, because 
no multi-cultural skills are gained, efficient university quality comes at the expense of inefficiently low multi-cultural skills. A reversed inefficiency can be observed in the other extreme where students are fully mobile. In this case, students acquire an efficient level of multi-cultural skills, but university quality is generally inefficient because the countries do not internalize the externalities between them. Thus, we do not find a mobility level that induces both efficient quality and an efficient acquisition of multi-cultural skills. As a consequence, optimal mobility levels will have to trade-off inefficiencies in university quality against inefficiencies in multi-cultural skills.

The direction in which student mobility distorts university quality depends on the relative strength of a positive competition and a negative free rider effect. If the free rider effect outweighs the competition effect, the quality of university education will be inefficiently low and vice versa.

Higher student mobility increases the relative strength of the free rider effect in its relation to the competition effect. As student mobility increases, the talent pool of mobile students decreases. Due to this composition effect, a country's incentives to attract foreign students declines. At the same time, higher mobility also lowers the average talent of immobile students. This composition effect weakens a country's incentive to retain native students. Hence, both composition effects strengthen the free rider effect at the expense of the competition effect. As a consequence we obtain that only if the initial competition effect is particularly strong, compared to the initial free rider effect, university quality exceeds efficient levels. Thus we conclude that, at an optimal second best level of student mobility, an underprovision of university quality is more likely to occur.

\section{Appendix}

Proof of Proposition 1: We solve the maximization problem $\max _{q_{A}, q_{B}} W\left(q_{A}, q_{B}\right)$. The function $W\left(q_{A}, q_{B}\right)$ is continuous, but, due to corner conditions, consists of three parts. Whenever $\left|q_{A}-q_{B}\right| \leq m / 2$ we have

$$
\begin{aligned}
W\left(q_{A}, q_{B}\right)= & {\left[4 m\left(q_{A}-2 q_{A}\left(k_{2}+k_{1} q_{A}\right)+q_{B}-2 q_{B}\left(k_{2}+k_{1} q_{B}\right)\right)\right.} \\
& \left.+m^{2}(1-\hat{\tau})+4\left(q_{A}-q_{B}\right)^{2}\left(1-\hat{\tau}^{2}-4 k_{2}(1-\hat{\tau})\right)\right] /(8 m) .
\end{aligned}
$$


Since $W$ is concave in $q_{A}, q_{B}$, first order conditions are sufficient and yield $q_{A}=q_{B}=\left(1-2 k_{2}\right) /\left(4 k_{1}\right)$.

Whenever $q_{A}-q_{B}>m / 2$ we have $a_{A}^{0}=-1 / 2$ and $a_{B}^{0}=1 / 2$ so that

$$
\begin{aligned}
W\left(q_{A}, q_{B}\right)= & {\left[2 m\left(q_{B}-k_{1}\left(q_{A}^{2}+q_{B}^{2}\right)+\left(q_{A}-q_{B}\right) \hat{\tau}^{2}\right)\right.} \\
& -\left(2 k_{2}\left(m\left(q_{A}+q_{B}\right)+2\left(q_{A}-q_{B}\right)^{2}(1-\hat{\tau})\right] /(2 m)\right.
\end{aligned}
$$

This expression is concave in $\left(q_{A}, q_{B}\right)$ so that first order conditions are sufficient. First order condition yield $q_{A}-q_{B}=-m\left(1-\hat{\tau}^{2}\right) /\left(2 k_{1} m+8 k_{2}(1-\hat{\tau})<\right.$ 0 , which violates $q_{A}-q_{B}>m / 2$. Hence, there is no internal maximum for $W$ on the domain $q_{A}-q_{B}>m / 2$.

Whenever $q_{A}-q_{B}<-m / 2$ we have $a_{A}^{0}=1 / 2$ and $a_{B}^{0}=-1 / 2$ so that

$$
\begin{aligned}
W\left(q_{A}, q_{B}\right)= & {\left[2 m\left(q_{A}\left(1-k_{2}-k_{1} q_{A}\right)-k_{2} q_{B}-k_{1} q_{B}^{2}\right)\right.} \\
& \left.-4 k_{2}\left(q_{A}-q_{B}\right)^{2}(1-\hat{\tau})-m\left(q_{A}-q_{B}\right) \hat{\tau}^{2}\right] /(2 m)
\end{aligned}
$$

This expression is concave in $\left(q_{A}, q_{B}\right)$ so that first order conditions are sufficient. First order condition yield $q_{A}-q_{B}=m\left(1-\hat{\tau}^{2}\right) /\left(2 k_{1} m+8 k_{2}(1-\hat{\tau})>0\right.$, which violates $q_{A}-q_{B}<-m / 2$. Hence, there is no internal maximum for $W$ on the domain $q_{A}-q_{B}<-m / 2$.

Since $W\left(q_{A}, q_{B}\right)$ is continuous over its entire domain, the maximum is unique and obtained for

$$
q_{A}=q_{B}=\frac{1-2 k_{2}}{4 k_{1}}
$$

Q.E.D.

Proof of Proposition 2: Substitution of $q_{A}=q_{B}=q_{E}$ into $W\left(q_{A}, q_{B}\right)$ and a rearrangement of terms yields

$$
W\left(q_{E}, q_{E}\right)=\frac{m k_{1}\left(1-\hat{\tau}^{2}\right)+\left(1-2 k_{2}\right)^{2}}{8 k_{1}} .
$$

For $m=0$ this expression is independent of $\hat{\tau}$. For $m>0$ the expression is strictly decreasing for $\hat{\tau}>0$. Hence, $\hat{\tau}=0$ is optimal.

Q.E.D.

Proof of Proposition 3: Follows directly from Proposition 1 and 2. Q.E.D.

Proof of Lemma 1: The pair $\left(q_{A}^{*}, q_{B}^{*}\right)$ is a Nash equilibrium whenever

$$
q_{A}^{*}=\arg \max _{q_{A}} W_{A}\left(q_{A}, q_{B}^{*}\right) \text { and } q_{B}^{*}=\arg \max _{q_{B}} W_{B}\left(q_{A}^{*}, q_{B}\right) .
$$


Whenever $k_{1}>1 / m$ and $k_{2}<1 / 4$ the solution, for $\left|q_{A}-q_{B}\right| \leq m / 2$, is

$$
q_{A}=q_{B}=q^{*}=\frac{\left(1-4 k_{2}+\hat{\tau}^{2}+\left(1-\hat{\tau}^{2}\right) E\right) m}{8 m k_{1}+8 k_{2}(1-\hat{\tau})-4\left(1-\hat{\tau}^{2}\right) E},
$$

which is consistent with the assumption $\left|q_{A}-q_{B}\right| \leq m / 2$.

We now show that $q_{A}<q_{B}-m / 2$ is not a best response to $q_{B}=q^{*}$. For $q_{A}<q_{B}-m / 2$ the social welfare of country $\mathrm{A}$ is

$$
W^{A}=W_{1}^{A} \equiv \hat{\tau}^{2} q_{A} / 2+(1-E)\left(1-\hat{\tau}^{2}\right) q_{B} / 2-k_{1} q_{A}^{2}-k_{2} \hat{\tau} q_{A} .
$$

Not that for the range $q_{A} \in\left[0, q_{B}-m / 2\right]$ the expression

$$
\partial W_{1}^{A} / \partial q_{A}=\hat{\tau}^{2} / 2-2 k_{1} q_{A}-k_{2} \hat{\tau}
$$

is maximized for $q_{A}=q_{B}-m / 2$. For $q_{B}=q^{*}$ it then follows

$$
\frac{\partial W_{1}^{A}}{\partial q_{A}}=\hat{\tau}^{2} / 2-k_{2} \hat{\tau}-2 k_{1}\left(\frac{m\left(1+E-4 k_{2}+\hat{\tau}^{2}(1-E)\right)}{8\left(k_{2}(1-\hat{\tau})+k_{1} m\right)-4 E\left(1-\hat{\tau}^{2}\right)}-\frac{m}{2}\right) .
$$

This expression is decreasing in $E$ so that the expression is smallest for $E=1$ :

$$
\frac{\partial W_{1}^{A}}{\partial q_{A}}=\hat{\tau}^{2} / 2-k_{2} \hat{\tau}-2 k_{1}\left(\frac{m\left(2-4 k_{2}\right)}{8\left(k_{2}(1-\hat{\tau})+k_{1} m\right)-4\left(1-\hat{\tau}^{2}\right)}-\frac{m}{2}\right) .
$$

For $k_{1}>1 / m$ and $k_{2}<1 / 4$ the expression is positive. This shows that $W^{A}\left(q_{A}, q^{*}\right)$ is increasing on $q_{A} \in\left[0, q^{*}-m / 2\right)$ so that $q_{A} \in\left[0, q^{*}-m / 2\right)$ is not a best response to $q_{B}=q^{*}$.

Next we show that also $q_{A}>q_{B}+m / 2$ is not a best response to $q_{B}=q^{*}$. For $q_{A}>q_{B}+m / 2$ it follows

$$
W^{A}=W_{2}^{A} \equiv q_{A}\left(1-4 k_{2}+E\left(1-\hat{\tau}^{2}\right)-2 k_{1} q_{A}+2 k_{2} \hat{\tau}\right) / 2
$$

Hence,

$$
\frac{\partial W_{2}^{A}}{\partial q_{A}}=\left(1-4 k_{2}+E\left(1-\hat{\tau}^{2}\right)-4 k_{1} q_{A}+2 k_{2} \hat{\tau}\right) / 2
$$

for $q_{A}>q_{B}+m / 2$. For $k_{1}>1 / m, k_{2}<1 / 4$, and $q_{B}=q^{*}$ it may be shown that expression (4) evaluated at $q_{A}=q^{*}+m / 2$ is negative. From this and the fact that (4) is decreasing in $q_{A}$ it follows that $W^{A}\left(q_{A}, q^{*}\right)$ is decreasing for $q_{A}>q^{*}+m / 2$ so that $q_{A}>q^{*}+m / 2$ is not a best response to $q_{B}=q^{*}$. 
It follows that $q_{A}=q^{*}$ is a best response to $q_{B}=q^{*}$. Due to symmetry $q_{B}=q^{*}$ is also a best response to $q_{A}=q^{*}$. Therefore $q_{A}=q_{B}=q^{*}$ is the unique symmetric equilibrium. Q.E.D.

Proof of Proposition 4: Substitution of $\hat{\tau}=0$ in equation (3) and its comparison to $q_{E}$ yields the result.

Q.E.D.

Proof of Lemma 2: Taking the derivative of (3) with respect to $\hat{\tau}$ yields

$$
\frac{d q^{*}}{d \hat{\tau}}=\frac{k_{2}\left(1-4 k_{2}+E\right)+2\left(\left(m k_{1}+k_{2}\right)(1-E)-E\right) \hat{\tau}-k_{2}(1-E) \hat{\tau}^{2}}{2\left(2\left(m k_{1}+(1-\hat{\tau}) k_{2}\right)-E\left(1-\hat{\tau}^{2}\right)\right)^{2} / m} .
$$

Since the denominator of (5) is strictly positive, this derivative is continuous for all $\hat{\tau} \in[0,1]$. Moreover, the numerator determines its sign. Because the numerator is quadratic in $\hat{\tau}$ with a negative coefficient $-k_{2}(1-E)<0$, the sign of $d q^{*} / d \hat{\tau}$ changes at most once on $\hat{\tau} \in[0,1]$. Due to $k_{2}<1 / 4$, the numerator is positive at $\hat{\tau}=0$. Hence, the sign changes if and only if the numerator in (5) evaluated at $\hat{\tau}=1$ is negative. That is whenever

$$
E>\frac{m k_{1}+k_{2}\left(1-2 k_{2}\right)}{m k_{1}+1-2 k_{2}}
$$

Q.E.D.

Proof of Proposition 5: After a substitution of the Nash values (3) into the aggregate social welfare function (2), we may rewrite the change in welfare as

$$
\frac{d W}{d \hat{\tau}}=\frac{d q(\hat{\tau})}{d \hat{\tau}}\left[q_{E}-q(\hat{\tau})\right]-\frac{\hat{\tau} m}{4} .
$$

Since $q(1)=q_{E}$ the evaluation of the derivative at $\hat{\tau}=1$ yields $-m / 4$. Hence, aggregate social welfare is decreasing at $\hat{\tau}=1$. The derivative evaluated at $\hat{\tau}=0$ yields

$$
\frac{\left(1-4 k_{2}+E\right) k_{2} m\left[(1-E) k_{1} m+\left(2 k_{2}-E\right)\left(1-2 k_{2}\right)\right]}{2\left(2\left(k_{2}+k_{1} m\right)-E\right)^{3}}
$$

This expression is positive if and only if $E<E_{H}$. Hence, for $E<E_{L}<E_{H}$, aggregate social welfare is increasing at $\hat{\tau}=0$ and decreasing at $\hat{\tau}=1 \mathrm{It}$ follows for the optimal degree of mobility that $\hat{\tau}^{*} \in(0,1)$. From Proposition 3 and Lemma 3 it follows that there is an underprovision of quality. Q.E.D. 
Proof of Lemma 3: The statement is a corollary of the proof of Lemma 2. Q.E.D.

Proof of Lemma 4: In the proof of Lemma 2 we established that $\partial q^{*} / \partial \hat{\tau}$ is continuous on $\hat{\tau} \in[0,1]$, changes sign at most once, and has at most one maximum. Moreover, the proof of Lemma 2 established that $\partial q^{*} / \partial \hat{\tau}$ at $\hat{\tau}=0$ is positive, so that $q^{*}$ is increasing at $\hat{\tau}=0$.

Evaluation of $\partial q^{*} / \partial \hat{\tau}$ at $\hat{\tau}=1$ yields

$$
\left.\frac{\partial q^{*}}{\partial \hat{\tau}}\right|_{\hat{\tau}=1}=\frac{(1-E) k_{1} m+\left(1-2 k_{2}\right)\left(k_{2}-E\right)}{4 k_{1}^{2} m},
$$

which is negative for $E>E_{L}$. Hence, $q^{*}$ is decreasing at $\hat{\tau}=1$.

Since $q^{*}$ at $\hat{\tau}=0$ exceeds $q_{E}$ and, due to Proposition $4, q^{*}$ is smaller than $q_{E}$ at $\hat{\tau}=1$, continuity of $\partial q^{*} / \partial \hat{\tau}$ and the fact that it changes sign only once imply that there exists a unique $\bar{\tau} \in(0,1)$ such that $q^{*}(\bar{\tau})=q_{E}$. $\quad$ Q.E.D.

Proof of Proposition 6: By Lemma 4 there exists a unique $\bar{\tau} \in(0,1)$ such that $q(\hat{\tau})<q_{E}$ for all $\hat{\tau} \in(0, \bar{\tau})$ and $q(\hat{\tau})>q_{E}$ for all $\hat{\tau} \in(\bar{\tau}, 1)$. Since $q^{*}(1)=q_{E}$ the continuity of $q(\hat{\tau})$ implies that there exists a maximum $q(\tilde{\tau})>q_{E}$ at some $\tilde{\tau} \in(\bar{\tau}, 1)$. We first establish that $\hat{\tau}>\tilde{\tau}$ cannot be optimal. To see this, observe there exists a $\tau^{\prime} \in[\bar{\tau}, \tilde{\tau})$ such that $q\left(\tau^{\prime}\right)=q(\hat{\tau})$. Consequently, the difference in aggregate welfare can be expressed as

$$
W(\hat{\tau})-W\left(\tau^{\prime}\right)=\int_{\tau^{\prime}}^{\hat{\tau}} \frac{d W}{d \hat{\tau}} d \tau=\int_{\tau^{\prime}}^{\hat{\tau}} \frac{\partial W}{\partial \hat{\tau}} d \tau=\int_{\tau^{\prime}}^{\hat{\tau}}-\frac{m \tau}{4} d \tau<0,
$$

where the second equality follows because $q\left(\tau^{\prime}\right)=q(\hat{\tau})$. Hence, $\hat{\tau}>\tilde{\tau}$ is not optimal.

Second we establish that $\hat{\tau} \in[\bar{\tau}, \tilde{\tau})$ cannot be optimal. This follows from the observation (6) that

$$
\frac{d W}{d \hat{\tau}}=\frac{d q}{d \hat{\tau}}\left[q_{E}-q(\hat{\tau})\right]-\hat{\tau} / 4 .
$$

Since for $\hat{\tau} \in[\bar{\tau}, \tilde{\tau})$ we have $d q / d \hat{\tau}<0$ and $\left.q(\hat{\tau})>q_{E}\right)$ the expression $d W / d \hat{\tau}$ is negative for any $\hat{\tau} \in[\bar{\tau}, \tilde{\tau})$.

Consequently, the second best optimum is characterized by some $\hat{\tau} \in$ $(0, \bar{\tau})$. As for $\hat{\tau}<\bar{\tau}$, it follows $q(\hat{\tau})<q_{E}$, there is underprovision of quality. Q.E.D. 
Proof of Lemma 5: All but the last statement follow directly from the proof of Lemma 4 . The last statement follows from the fact that $q^{*}(\tilde{\tau})>$ $q^{*}(0)>q_{E}=q^{*}(1)$. Hence, continuity of $q^{*}($.$) implies existence of \tau^{\prime} \in(\tilde{\tau}, 1)$ such that $q^{*}\left(\tau^{\prime}\right)=q^{*}(0)$.

Proof of Proposition 7: For any $\hat{\tau} \in\left(\tilde{\tau}, \tau^{\prime}\right)$ there exists a $\tau \in(0, \tilde{\tau})$ with $q(\tau)=q(\hat{\tau})$. Consequently, the difference in aggregate welfare can be expressed as

$$
W(\hat{\tau})-W(\tau)=\int_{\tau}^{\hat{\tau}} \frac{d W}{d \hat{\tau}}(t) d t=\int_{\tau}^{\hat{\tau}} \frac{\partial W}{\partial \hat{\tau}}(t) d \tau=\int_{\tau}^{\hat{\tau}}-\frac{m \tau}{4} d \tau<0,
$$

where the second equality follows because $q(\tau)=q(\hat{\tau})$. Hence, $\hat{\tau} \in\left(\tilde{\tau}, \tau^{\prime}\right)$ is not optimal.

Equation (6) implies that whenever $q^{*}(\tau)>q_{E}$ and $q^{*}(\tau)$ is decreasing, then aggregate welfare $W(\hat{\tau})$ is decreasing in $\hat{\tau}$. Consequently, $\hat{\tau} \in(0, \tilde{\tau})$ cannot be optimal.

Hence, the second best $\hat{\tau}$ lies either in the interval $\left(\tau^{\prime}, 1\right)$ or at 0. Q.E.D.

\section{References}

M. Beine, F. Docquier and H. Rapoport. (2001). Brain Drain and Economic Growth: Theory and Evidence. Journal of Development Economics 64, 275289.

J. Bhagwati and K. Hamada. (1974). The Brain Drain, International Integration of Markets for Professionals and Unemployment. Journal of Development Economics 64, 275-289.

T. Büttner and R. Schwager. (2004). Regionale Verteilungseffekte der Hochschulfinanzierung und ihre Konsequenzen. In: Franz, W., Ramser, H. J., Stadler, M., Bildung, 33. Wirtschaftswissenschaftliches Seminar Ottobeuren, Tübingen, forthcoming.

H. B. Grubel and A. D. Scott. (1966). The International Flow of Human Capital. American Economic Review 56, 268-274.

V. Jahr, H. Schomburg and U. Teichler. (2002). Internationale Mobilität von Absolventinnen und Absolventen europäischer Hochschulen. Werkstattberichte des Wissenschaftlichen Zentrums für Berufs- und Hochschulforschung der Universität Kassel, Vol. 61. 
M. Justman and J.-F. Thisse. (1997). Implications of the mobility of skilled labor for local public funding of higher education. Economic Letters $55,409-412$.

A. Kemnitz. (2005). Educational Federalism and the Quality Effects of Tuition Fees. mimeo.

P. Poutvaara. (2004a). Educating Europe: Should Public Education be Financed with Graduate Taxes or Income-Contingent Loans? CESifo Economic Studies 50 (4), 663-684.

P. Poutvaara. (2004b). Public Education in an Integrated Europe: Studying to Migrate and Teaching to Stay? CESifo Working Paper No. 1369.

O. Stark, C. Helmenstein and A. Prskawetz. (1997). A Brain Gain with a Brain Drain. Economic Letters 55, 227-234.

O. Stark and Y. Wang. (2002). Inducing Human Capital Formation: Migration as a Substitute for Subsidies. Journal of Public Economics 86, $29-46$. 


\section{SFB 649 Discussion Paper Series 2006}

For a complete list of Discussion Papers published by the SFB 649, please visit http://sfb649.wiwi.hu-berlin.de.

001 "Calibration Risk for Exotic Options" by Kai Detlefsen and Wolfgang K. Härdle, January 2006.

002 "Calibration Design of Implied Volatility Surfaces" by Kai Detlefsen and Wolfgang K. Härdle, January 2006.

003 "On the Appropriateness of Inappropriate VaR Models" by Wolfgang Härdle, Zdeněk Hlávka and Gerhard Stahl, January 2006.

004 "Regional Labor Markets, Network Externalities and Migration: The Case of German Reunification" by Harald Uhlig, January/February 2006.

005 "British Interest Rate Convergence between the US and Europe: A Recursive Cointegration Analysis" by Enzo Weber, January 2006.

006 "A Combined Approach for Segment-Specific Analysis of Market Basket Data" by Yasemin Boztuğ and Thomas Reutterer, January 2006.

007 "Robust utility maximization in a stochastic factor model" by Daniel Hernández-Hernández and Alexander Schied, January 2006.

008 "Economic Growth of Agglomerations and Geographic Concentration of Industries - Evidence for Germany" by Kurt Geppert, Martin Gornig and Axel Werwatz, January 2006.

009 "Institutions, Bargaining Power and Labor Shares" by Benjamin Bental and Dominique Demougin, January 2006.

010 "Common Functional Principal Components" by Michal Benko, Wolfgang Härdle and Alois Kneip, Jauary 2006.

011 "VAR Modeling for Dynamic Semiparametric Factors of Volatility Strings" by Ralf Brüggemann, Wolfgang Härdle, Julius Mungo and Carsten Trenkler, February 2006.

012 "Bootstrapping Systems Cointegration Tests with a Prior Adjustment for Deterministic Terms" by Carsten Trenkler, February 2006.

013 "Penalties and Optimality in Financial Contracts: Taking Stock" by Michel A. Robe, Eva-Maria Steiger and Pierre-Armand Michel, February 2006.

014 "Core Labour Standards and FDI: Friends or Foes? The Case of Child Labour" by Sebastian Braun, February 2006.

015 "Graphical Data Representation in Bankruptcy Analysis" by Wolfgang Härdle, Rouslan Moro and Dorothea Schäfer, February 2006.

016 "Fiscal Policy Effects in the European Union" by Andreas Thams, February 2006.

017 "Estimation with the Nested Logit Model: Specifications and Software Particularities" by Nadja Silberhorn, Yasemin Boztuğ and Lutz Hildebrandt, March 2006.

018 "The Bologna Process: How student mobility affects multi-cultural skills and educational quality" by Lydia Mechtenberg and Roland Strausz, March 2006.

\section{SFB 649, Spandauer Straße 1, D-10178 Berlin} http:/ / sfb649.wiwi.hu-berlin.de 